\title{
Escape and absconding among offenders with schizophrenia spectrum disorder - an explorative analysis of characteristics
}

\author{
Johannes Kirchebner ${ }^{*}$, Steffen Lau and Martina Sonnweber
}

\begin{abstract}
Background: Escape and absconding, especially in forensic settings, can have serious consequences for patients, staff and institutions. Several characteristics of affected patients could be identified so far, albeit based on heterogeneous patient populations, a limited number of possible factors and basal statistical analyses. The aim of this study was to determine the most important characteristics among a large number of possible variables and to describe the best statistical model using machine learning in a homogeneous group of offender patients with schizophrenia spectrum disorder.

Methods: A database of 370 offender patients suffering from schizophrenia spectrum disorder and 507 possible predictor variables was explored by machine learning. To counteract overfitting, the database was divided into training and validation set and a nested validation procedure was used on the training set. The best model was tested on the validation set and the most important variables were extracted.

Results: The final model resulted in a balanced accuracy of $71.1 \%(95 \% \mathrm{Cl}=[58.5,83.1])$ and an AUC of 0.75 $(95 \% \mathrm{Cl}=[0.63,0.87])$. The variables identified as relevant and related to absconding/ escape listed from most important to least important were: more frequent forbidden intake of drugs during current hospitalization, more index offences, higher neuroleptic medication, more frequent rule breaking behavior during current hospitalization, higher PANSS Score at discharge, lower age at admission, more frequent dissocial behavior during current hospitalization, shorter time spent in current hospitalization and higher PANSS Score at admission.
\end{abstract}

Conclusions: For the first time a detailed statistical model could be built for this topic. The results indicate the presence of a particularly problematic subgroup within the group of offenders with schizophrenic spectrum disorder who also tend to escape or abscond. Early identification and tailored treatment of these patients could be of clinical benefit.

Keywords: Absconding, Escape, Schizophrenia, Offending, Machine learning, Forensic psychiatry

\footnotetext{
* Correspondence: johannes.kirchebner@puk.zh.ch

Department of Forensic Psychiatry, University Hospital of Psychiatry Zurich,

Zurich, Switzerland
}

C C The Author(s). 2021 Open Access This article is licensed under a Creative Commons Attribution 4.0 International License, which permits use, sharing, adaptation, distribution and reproduction in any medium or format, as long as you give appropriate credit to the original author(s) and the source, provide a link to the Creative Commons licence, and indicate if changes were made. The images or other third party material in this article are included in the article's Creative Commons licence, unless indicated otherwise in a credit line to the material. If material is not included in the article's Creative Commons licence and your intended use is not permitted by statutory regulation or exceeds the permitted use, you will need to obtain permission directly from the copyright holder. To view a copy of this licence, visit http://creativecommons.org/licenses/by/4.0/ The Creative Commons Public Domain Dedication waiver (http://creativecommons.org/publicdomain/zero/1.0/) applies to the data made available in this article, unless otherwise stated in a credit line to the data. 


\section{Background}

In the mental health system, escape is defined as gaining freedom by breaking through the secured perimeter, in forensic psychiatric hospitals often the outer wall or fence of a ward [1,2], while absconding is defined as gaining freedom by evading the supervision of staff during a controlled opening outside the ward or hospital [1]. As forensic psychiatry is defined as "a subspecialty of psychiatry in which scientific and clinical expertise is applied in legal contexts involving civil, criminal, correctional, regulatory, or legislative matters, and in specialized clinical consultations in areas such as risk assessment or employment" this is a crucial area of research and daily clinical practice in this field.

Both escape and absconding from closed psychiatric wards, and especially from secure forensic hospitals, can be serious events leading to potentially grave outcomes [1-5]. These can directly affect the patient concerned, such as overdosing [6-8], self-inflicted injury [8-10], attempted suicide [10-14] or completed suicide [15, 16], prolonged rehabilitation and treatment [17], medication non-compliance [17], violence against others $[4,9,14]$ or (re-) offending [8, 13, 15, 18-22].

The reputation of institution and staff can also be damaged by such incidents and subsequent negative media coverage regarding mental health and security may lead to increased stigma, reduced confidence in the particular institution or the health care system as a whole, anger and guilt among staff, stress and anxiety among patients [4, 23-25].

Despite those detrimental effects, research on this topic is rather sparse $[1,2]$. Recent reviews have identified 39 studies on escape and absconding in general psychiatry $[2,17]$, but only nine on the subject in forensic psychiatry, although the consequences are particularly severe in the latter setting [23].

Most of the relevant research on escape and absconding was conducted by Bowers [9, 26-28], who focused primarily on the analysis of the characteristics of affected patients. Subsequent studies on this topic were based on these characteristics and were predominantly undertaken in the United Kingdom and Australia. The diagnosis of schizophrenia spectrum disorder (SSD) has been identified as one of the most influential characteristics for escape or absconding with incidences of up to $71 \%$ in patients examined $[1,4,5,17,23,24,27-33]$. Further risk factors for escape or absconding included: younger age $[1,3-5,9,29,33-36]$, male sex $[1,4,5,29,30,33$, 36-38], being unemployed [4, 37, 39], being unmarried $[1,5,7,10,36,39]$, homelessness [38], the number of diagnoses [38], having a personality disorder $[1,4,5,11]$ or affective disorder $[4,5,9,33,36]$, prior convictions [4, 17, 19], forensic psychiatric treatment in the past [1], absconding in the past $[1,5,23,24,30,32,35,39]$, compulsory detainment $[1,5,38]$, treatment noncompliance [1] and medication non-compliance in the past [5], exacerbation of symptoms [19, 28, 31], aggressive behavior [24, 38], alcohol or drug abuse [1, 4, 5, 23, $24,33,38-40]$, a history of sexual abuse [1], not having a history of self-inflicted harm [38], suicidal behavior [23], shorter duration of current hospitalization [3, 4, 33, $34,38,41]$ and also longer duration of current hospitalization [23, 30].

Although past research has provided important insights into the topic, certain weaknesses are apparent: (1) Previous studies included heterogeneous patient populations with different diagnoses. Although individuals diagnosed with SSD constitute the majority of escapees and absconders, there is no study to date that has examined these patients exclusively (2). The investigated features are the result of the valuable work of Bowers and his colleagues and were reproduced in subsequent studies. However, new and potentially more specific factors could not be uncovered so far. (3) The statistical analyses of earlier studies were mostly based on null hypothesis significance testing (NHST), in rare cases on linear regression methods, which was already recognized as a problem in 2000 [42], yet received only limited attention in further studies. The problem with examining multiple single variables with NHST, known as multiple testing $[43,44]$, is that each statistical test is associated with a false positive rate (type I error) and continues to accumulate with each additional test. This results in variables being falsely identified as significant and is further exacerbated when there is no correction for multiple testing $[45,46]$. Another limitation of the NHST is the insufficient consideration of interactions between the variables and that no evaluations of the quality of statistical models are provided. Simply looking at the significant variables may produce models and interpretations of limited informative value. Therefore, the aim of this study is to investigate the phenomenon of escape and absconding employing a complex data set of 370 offenders with SSD and over 500 potential influential variables via machine learning.

\section{Machine learning}

Due to technical and scientific progress in the fields of mathematics and computer science, it is now possible to perform statistical calculations and pattern recognition using complex statistical algorithms. In this context, the term machine learning (ML) is commonly used. The advantages of $\mathrm{ML}$ are manifold: large amounts of data can be processed quickly, a multitude of possible variables and their influence on each other can be investigated, complex nonlinear relationships can be calculated, and predictive models with different performance measures can be built and evaluated. This also appears to be a 
promising approach for new analyses in the medical sciences. In psychiatry, ML is already used in several areas, such as neuroimaging and clinical decision making. In the subfield of forensic psychiatry, its application is still relatively rare. However, in this area, where basic knowledge is somewhat scarce but extensive datasets may already exist, ML represents a promising opportunity to gain new insights - for example regarding the characteristics leading to escape or absconding (for more information on ML see [47-50]).

\section{Objectives}

Using ML algorithms, the aim of this exploratory study was to identify the most influential parameters in an extensive database distinguishing between patients who escape or abscond and those who do not, based on the unique group of forensic offender patients with SSD, and to quantify the performance of the calculated model.

\section{Methods \\ Setting}

Data was obtained in a single mental health facility, Switzerland's largest forensic psychiatric clinic specialized in the treatment of patients suffering from schizophrenia or other acute psychiatric pathology. The clinic holds a total of 79 beds and offers court mandated treatment (often for several years) for patients who have committed a crime or regular prisoners whose mental health status does not allow treatment within prison. Patients are treated in different levels of care and security according to treatment effort and reduced dangerousness. According to "The Matrix of Security" [51], 27 of the beds are within a high-security setting where no leaves are permitted. 39 beds are in closed wards with medium- to low-security settings, and 13 are in an open ward with low-security setting. Leaves are approved after a detailed assessment process (often reviewed by judicial authorities) that begins with short walks accompanied by professional staff in one-on-one supervision. If treatment leads to an improvement in psychopathology and reduces the risk of future offending, the patient may be allowed to leave the ward unaccompanied, initially for a short time, and later for a longer period, to expedite rehabilitation.

\section{Source of data and measures}

The files of 370 offender patients diagnosed with SSD as defined in ICD-9 [52] or ICD-10 [53] were analyzed retrospectively. The coding protocol covered the following domains: social-demographic data, childhood/ youth experiences, psychiatric history, past criminal history, social and sexual functioning, details on the offence leading to forensic hospitalization, prison data, particularities of the current hospitalization and psychopathological symptoms by closely adopting the positive and negative symptom scale (PANSS), whereby symptoms were divided into the usual 30 sub-categories and rated on a scale (completely absent, discretely present or substantially present). Our extensive database has already been used in other studies and is part of a larger project in which the medical records of forensic inpatients have been analyzed in detail to obtain insights into the underresearched area of SSD and criminal behavior. Full details on data collection and processing can be found in Kirchebner et al. [54] and Kirchebner et al. [55].

\section{Statistical procedures - machine learning}

Parts of the following section were published in Günther et al. [51] and are partially adopted here and extended to include the methodology of the current research question. Given the explorative nature of this study, supervised machine learning (ML) appeared to be the optimal method to identify the most important influencing factors of a variety of variables and to determine the model providing the best predictive power. An Overview of the statistical steps can be seen in Fig. 1 and are further described below. All Steps were performed using $\mathrm{R}$ version 3.6.3. and the MLR package v2.171 [56]. CI calculations of the balanced accuracy were conducted using MATL AB R2019a (MATLAB and Statistics Toolbox Release 2012b, The MathWorks, Inc., Natick, Massachusetts, United States) with the add-on "computing the posterior balanced accuracy” v1.0 [57].

\section{Preliminary data processing and measures}

All raw data was first prepared for machine learning (see Fig. 1 Step 1) - multiple categorical variables were transformed to binary code. Continuous and ordinal variables were not adjusted. Owing to the retrospective nature of the study and the large number of variables collected, there were missing values, especially for information on patients' in-depth biographical history. Variables with more than $33 \%$ missing values were eliminated resulting in a remaining set of 508 variables.

The outcome variable - patient, who escaped or absconded - was dichotomized into (1) patient escaped/ absconded and (2) patient did not escape/ abscond. The following incidents were considered as an event of absconding: running away from a member of staff or refusing to return to the unit with a member of staff, whilst on escorted leave or failure to return from unescorted leave. Escape was defined as successful or unsuccessful attempt to flee from the perimeter of the hospital.

Patients who were hospitalized in the high security area of our forensic hospital were excluded from the study because no (accompanied) leaves are permitted in this phase of treatment and no escape has ever occurred 


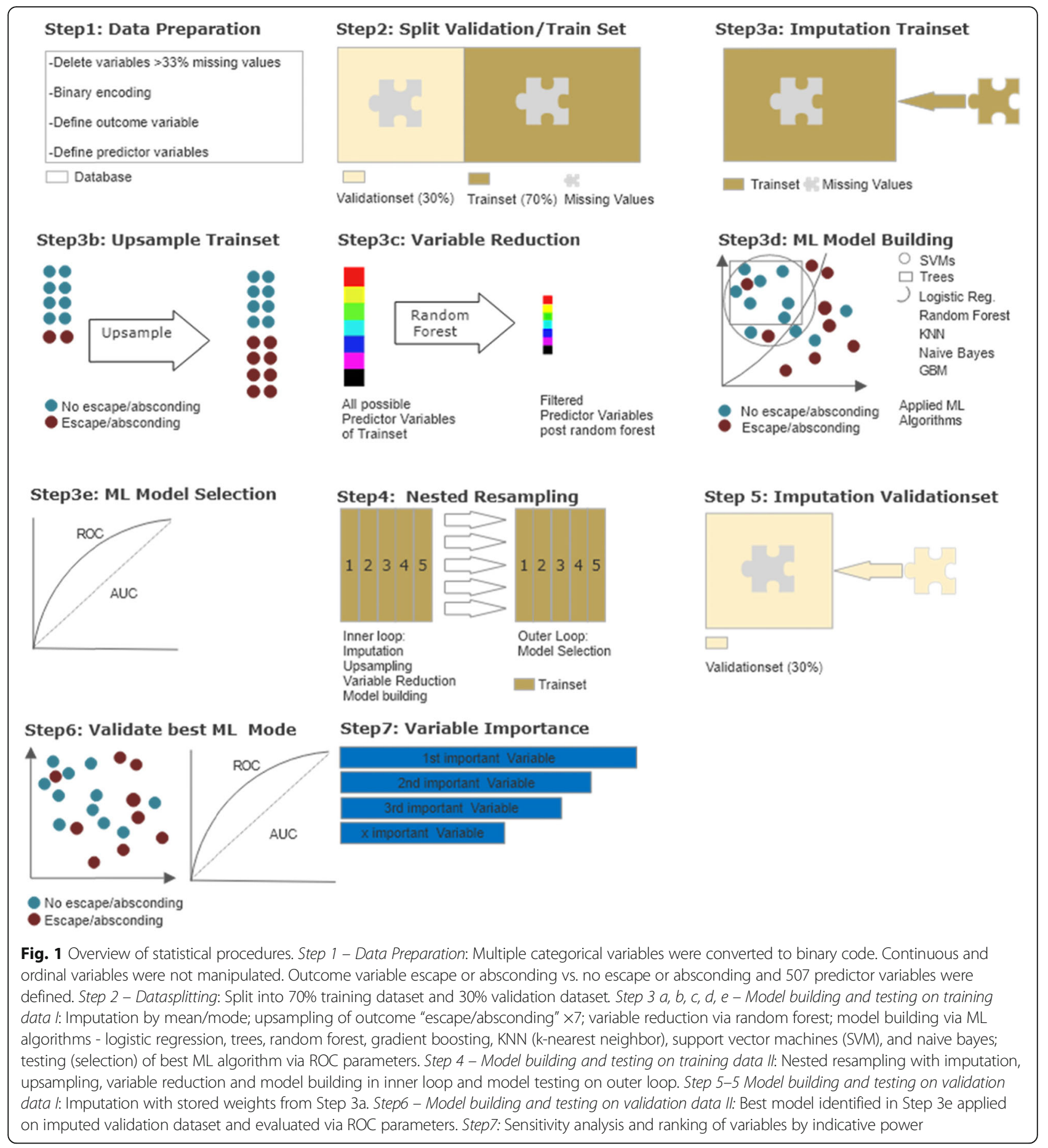

from this area. 274 patients remained for further analysis. Of these patients, 34 (12.4\%) were involved in an event of escape or absconding and 240 (87.6\%) were not. Non-escape/ absconding was defined as the positive class, escape/ absconding as the negative class.

The initial dataset was randomly divided into two subsets (see Fig. 1, Step 2) - a training dataset with $70 \%$ of all patient cases (191 patients) and a validation dataset with $30 \%$ of cases (83 patients). The training data set was utilized for variable reduction and model building/ selection (see Fig. 1, Steps 3x and 4) while the validation data set was applied to evaluate the prior selected statistical model (see Fig. 1, Step 5, 6 and 7). Predictor variable selection, model building and model evaluation were based on different subsets of the existing data to reduce the risk for overfitting. 
Imputation, balancing, variable filtering, statistical model building/ selection and nested resampling

All the subsequent steps under above section were conducted with the training data set (191 patients) only, while the data set for validation (83 patients) remained unchanged:

In order to flexibly apply all ML algorithms, imputation of missing values was performed. Imputation by mean for continuous variables and by mode for categorical variables included in the MLR package was applied (see Fig. 1, step 3a). The imputation weights were saved for later reuse on the validation dataset (see Fig. 1, step 5).

With a ratio of 12.4 to $87.6 \%$, the outcome variable was unevenly distributed. To create the most balanced result possible, random up sampling at a rate of 7 was used, leading to a more balanced result of 50.2 to $49.8 \%$ (see Fig. 1, Step 3b).

A main objective of the present study was to identify the most important predictor variables out of 507 possible variables. In addition, a reduction of variables can counteract overfitting and keep computation times at an acceptable level during initial model building. To this end, variable reduction to the 10 most important predictors was performed using the randomForestSRC package implemented in the MLR package, which evaluates variable importance [58] (see Fig. 1, step 3c).

Since our database of 274 observations is relatively small for machine learning purposes and we focused on variable extraction and prediction, we applied discriminative model building with logistic regression, trees, random forest, gradient boosting, KNN (k-nearest neighbor), support vector machines (SVM) and as an easily applicable generative model building, naive Bayes (for a more detailed description see [59]) (Fig. 1, Step 3d). No hyperparameters were optimized. The default hyperparameters can be obtained from the supplementary material.

The model performance of each model was calculated and assessed in terms of its balanced accuracy (the average of true positive and true negative rate, which is better suited for model evaluation and calculation of confidence intervals in imbalanced data [57]) and goodness of fit (measured with the receiver operating characteristic, balanced curve area under the curve method, ROC balanced AUC) [60]. Moreover, specificity, sensitivity, positive predictive value (PPV) and negative predictive value (NPV) were evaluated. As our training dataset was artificially balanced, the model with the highest AUC was chosen for final model validation on the validation dataset [60] (see Fig. 1, Step 3e).

Finally, the set of identified variables was tested for multicollinearity to avoid dependencies between the variables.
To avoid overfitting, it is advisable to include imputation, filtering, balancing and model building in a cross validation process kept separate from model-testing [61, 62].

Nested resampling seems best suited for this objective - in an inner loop data processing and model training are performed imbedded in cross-validation and then in an outer loop the performance of these models is tested also embedded in cross-validation. In this study the nested resampling model was built with the inner loop performing imputation, oversampling, variable filtering and model building within 5-fold cross-validation and the outer loop being used for performance evaluation also embedded in 5-fold-cross-validation, a technique of artificially creating different subsamples of a data set [63] (see Fig. 1, Step 4).

\section{Model validation and variable importance}

The validation subset of the total data (30\%, 83 patients) was imputed with the stored weights from Step 3a by mean and mode (see Fig. 1, Step 5a). Then, the best previously identified model was applied to the data and again the performance measures of this final model were assessed (see Fig. 1, Step 6). The variables used to predict the outcome variable (patient escaped/ absconded and patient did not escape/ abscond) in the final model were sorted by indicative power through means of a sensitivity analysis using the rminer package [64] (see Fig. 1, Step 7).

\section{Results}

An overview of the performance parameters of the different calculated models during the nested resampling procedure can be found in Table 1 . With a balanced accuracy of $76.7 \%,(95 \% \mathrm{CI}=[68.4,82.7])$ and an AUC of $0.88(95 \% \mathrm{CI}=[0.81,0.95])$ naïve bayes outperformed all other ML algorithms.

The quality of the final model in the validation step is shown in Table 2. As expected, the balanced accuracy of $71.1 \%(95 \% \mathrm{CI}=[58.5,83.1])$ and an AUC of $0.75(95 \%$ $\mathrm{CI}=[0.63,0.87])$ were less than the results of the initial training model, but still meaningful. With a sensitivity of $88.2 \%(95 \% \mathrm{CI}=[87.9,88.5])$ over three-quarters of patients who did not escape or abscond were identified correctly, with a specificity of $60 \%(95 \% \mathrm{CI}=[59.2$, $60.8]$ ), less than two-thirds of patients, who escaped or absconded were detected correctly.

The absolute and relative distribution of the 10 most influential variables among the whole dataset can be seen in Table 3. In addition to age at diagnosis, age at admission and number of index offences, the other influential variables covered circumstances related to the current hospitalization. Testing for multicollinearity showed no dependencies between the variables (detailed results see supplementary materials). 
Table 1 Machine learning models and performance in nested cross-validation on training dataset - escape/ absconding vs. no escape/ absconding

\begin{tabular}{|c|c|c|c|c|c|c|}
\hline Statistical Procedure & $\begin{array}{l}\text { Balanced } \\
\text { Acc. }(\%)(95 \% \mathrm{Cl})\end{array}$ & $\begin{array}{l}\text { AUC (\%) } \\
(95 \% \mathrm{Cl})\end{array}$ & $\begin{array}{l}\text { Sensitivity (\%) } \\
(95 \% \mathrm{Cl})\end{array}$ & $\begin{array}{l}\text { Specificity (\%) } \\
(95 \% \mathrm{Cl})\end{array}$ & $\begin{array}{l}\text { PPV (\%) } \\
(95 \% \mathrm{Cl})\end{array}$ & $\begin{array}{l}\text { NPV (\%) } \\
(95 \% \mathrm{Cl})\end{array}$ \\
\hline Logistic Regression & $73.9[64.7,79.8]$ & $0.81[0.72,0.89]$ & $80.2[76.0,84.4]$ & $67.6[52.5,82.7]$ & $95.8[93.5,98.1]$ & $26.9[17.9,35.9]$ \\
\hline Tree & $72.6[64.2,79.6]$ & $0.80[0.71,0.89]$ & $81.9[64.5,92.3]$ & $63.2[47.8,78.5]$ & $95.3[92.8,97.7]$ & $27.9[18.4,37.4]$ \\
\hline Random Forest & $71.1[61,76.6]$ & $0.84[0.76,0.92]$ & $94.8[92.4,97.1]$ & $47.4[31.5,63.2]$ & $94.2[91.8,96.7]$ & $50.0[33.7,66.3]$ \\
\hline Gradient Boosting & $65.8[58.4,73.8]$ & $0.82[0.74,0.90]$ & $89.5[86.3,92.8]$ & $42.1[26.4,57.8]$ & $93.3[90.6,96.0]$ & $30.8[18.2,43.3]$ \\
\hline KNN & $66.5[58.6,74.3]$ & $0.85[0.59,0.99]$ & $85.5[81.7,89.1]$ & $47.4[31.5,63.2]$ & $93.6[90.9,96.3]$ & $26.5[15.9,36.9]$ \\
\hline SVM & $73.4[65.5,80.9]$ & $0.84[0.76,0.92]$ & $94.2[91.7,96.7]$ & $52.6[36.8,68.5]$ & $94.7[92.3,97.1]$ & $50.0[34.5,65.5]$ \\
\hline Naive Bayes & $76.7[68.4,82.7]$ & $0.88[0.81,0.95]$ & $79.7[75.4,83.9]$ & $73.7[59.7,87.7]$ & $96.5[94.3,98.6]$ & $28.6[19.6,37.5]$ \\
\hline
\end{tabular}

AUC area under the curve (level of discrimination), PPV positive predictive value, NPV negative predictive value, KNN k-nearest neighbors, SVM support vector machines

A one-sided tornado graph comparing the relative importance of the identified variables during model validation is presented in Fig. 2. It shows the effect on the output variable (patient escaped/ absconded and patient did not escape/ abscond) by varying each predictor variable at a time, keeping all the other predictor variables at their initial values. The $\mathrm{x}$-axis represents the relative variable importance, the $y$-axis each variable - the wider the bar, the more impact the variable has on the model and the outcome. Consequently, the predictor variables are ranked from the most influential to the least influential.

\section{Discussion}

The aim of the study was to identify the most important characteristics of offender patients with SSD who escaped or absconded from a forensic hospital by means of ML and to describe the best possible statistical model. The identified variables were age at diagnosis of the SSD and age at admission, time spent in current hospitalization, problematic behavior during current hospitalization (e.g., forbidden intake of drugs/ alcohol, breaking of rules, dissocial behavior), amount of index offences, PANSS score at admission and discharge (note: if the patient did not return after the event of escape or absconding, the last PANSS before the event was taken) and dosing of neuroleptic medication (daily

Table 2 Final naïve bayes model performance measures on validation dataset - escape/ absconding vs. no escape/ absconding

\begin{tabular}{lll}
\hline Performance measures & \% & 95\% Confidence Interval \\
\hline Balanced Accuracy & 71.1 & {$[58.5,83.1]$} \\
AUC & 0.75 & {$[0.63,0.87]$} \\
Sensitivity & 88.2 & {$[87.9,88.5]$} \\
Specificity & 60.0 & {$[59.2,60.8]$} \\
PPV & 90.9 & {$[90.7,91.1]$} \\
NPV & 52.9 & {$[52.2,53.7]$} \\
\hline
\end{tabular}

cumulative olanzapine equivalent at discharge). The final model utilizing these variables achieved a balanced accuracy of $71 \%$ at an AUC of 0.75 with a sensitivity of $80 \%$ and a specificity of $60 \%$.

The escaped or absconded offender patients suffering from SSD shared several characteristics with escapees or absconders identified in previous studies in mixed populations. Our results confirm studies showing that a younger age plays a central role in escape or absconding [1, $3-5,9,27,29,33-36]$. Not only the age at the time of the incident was important as described in previous studies, but also a younger age at the time of the initial diagnosis of SSD. A shorter hospitalization period could also be confirmed $[3,4,31,33,34,38]$ indicating that escape and absconding arise in an earlier phase of treatment. Although, in contrast to previous investigations, no increased incidence of diagnoses of substance abuse was found $[1,4,5,23,24,33,38-40]$, there was a higher incidence of substance abuse during the current forensic hospitalization. Interestingly, this behavior was embedded in a general behavior of dissociality and a proneness to rule breaking. In this context, the newly identified variable "number of index offences" may be a possible indicator for an increased propensity to breach rules even before treatment. However, a difference in the frequency of diagnosis of dissocial personality disorder between the groups was not apparent, since diagnosing a personality disorder in the presence of SSD is excluded according to ICD coding.

Although the evidence on the relationship between escape or absconding and symptoms of SSD is scarce [25], previous studies have reported that symptoms were exacerbated at the time of escape or absconding [19, 28, 31]. Based on the objective scores of the PANSS over the course of treatment, our findings indicate that patients who escaped or absconded not only had an acute exacerbation of symptoms at that time, but they generally displayed a more severe course of the disorder. Presumably, this is also reflected in a higher demand for 
Table 3 Absolute and relative distribution of indicative variables on complete dataset - escape/ absconding vs. no escape/ absconding

\begin{tabular}{|c|c|c|c|c|}
\hline \multirow[t]{2}{*}{ Variable description } & \multicolumn{2}{|c|}{ Escape/absconding } & \multicolumn{2}{|c|}{ No escape/absconding } \\
\hline & $\mathrm{n} / \mathrm{N}(\%)$ & mean (SD) & $\mathrm{n} / \mathrm{N}(\%)$ & mean (SD) \\
\hline Age at admission & & $31.1(6.8)$ & & $34.1(10.2)$ \\
\hline Age at SSD diagnosis & & $24.4(5.6)$ & & $29.0(9.6)$ \\
\hline Dissocial behavoir reported during current hospitalisation & $25 / 34(73.5)$ & & $96 / 238(40.3)$ & \\
\hline Events of rule breaking during current hospitalization & $22 / 34(64.7)$ & & $50 / 236(21.2)$ & \\
\hline Amount of index offences & & $2.9(2.2)$ & & $2.1(1.7)$ \\
\hline Daily cumulative olanzapine equivalent at discharge ${ }^{a}$ & & $24.1(15.2)$ & & $18.6(14)$ \\
\hline Forbidden intake of drugs during current hospitalisation & $14 / 34(41.2)$ & & $11 / 240(4.6)$ & \\
\hline Time spent in current forensic hospitalization (in weeks) & & $140.7(126.8)$ & & $145.7(142.7)$ \\
\hline PANSS Score at admission & & $25.4(12.8)$ & & $24.3(12.4)$ \\
\hline PANSS Score at discharge & & $11.9(9.5)$ & & $11.4(10.1)$ \\
\hline
\end{tabular}

SD Standard deviation, PANSS positive and negative syndrome scale

aTo ensure comparability between different studies, daily neuroleptic doses were converted to olanzapine equivalents by using conversion factors derived from the classical weighted average dose method [65], the minimum effective dose method [66] or, in all other cases, from olanzapine equivalents based on international expert consensus [67]

neuroleptic drugs, which was found to be an influential factor in our sample. Our model did not identify any variables regarding childhood or adolescence and, with the exception of the two variables mentioned above, also no parameters from psychiatric or criminal history $[1,4$, $5,17,19,23,24,38]$. Hence, our analysis indicates that past patient information is less relevant than information on the current hospitalization.

In summary, we found that offender patients with SSD who escape or abscond tend to be younger, have a more severe level of pathology and are more likely to exhibit dissocial behavior.

In general research on populations of offenders with SSD, especially in the context of violence, age [68-74], dissocial patterns [68-75], substance abuse [75-77] and psychotic symptoms [78-85] have also been identified as risk factors and dominate the field of forensic research on SSD and offending. Escape and absconding are ruleviolating behaviors and thus perhaps a facet of dissocial behavior patterns that are interwoven with psychotic symptoms in a complex multifactorial framework that has not yet been sufficiently understood. It is possible that the group of offenders suffering from SSD comprises a subgroup of individuals who are particularly affected by these factors. For example, the framework by Hodgins' [69] postulates a subgroup ("early starters"), which is characterized by young age, dissocial and rule breaking behavior and drug abuse. They may also be more prone to problematic behavior during hospitalization and more likely to escape or abscond, which should be investigated in future studies.

Although the goal of the study was not to develop a tool to identify patients who flee or escape, the usefulness of the current model and subsequent opportunities for the potential development of such a tool need to be discussed. This will be illustrated by a cost matrix applied to the results of the validation data set. Patients correctly identified as non-absconders/ non-escapees (TP - 60 patients) or absconders/ escapees (TN - 9 patients) have no direct negative impact on the hospital and therefore generate zero cost. Patients who are incorrectly identified as Absconder/Escapees (FN - 8 patients) may receive more extensive treatment and be unnecessarily restrained. This results in costs of 20 units per patient. Patients who are misclassified as non-absconders/ non-escapees and then nonetheless escape (FP - 6 patients) pose a massive risk to society and themselves $[4$, 23-25]. Here we postulate a cost of 100 units per patient. Calculating the total cost for our current model results in: $60 \times 0+9 \times 0+8 \times 20+6 \times 100=760$ units. A perfect model with $100 \%$ specificity and sensitivity $(0$ FN/ 0 FP) results in no cost: $68 \times 0+15 \times 0+0 \times 20+$ $0 \times 100=0$ units. If the model correctly identified a patient who was misidentified as an absconder/escapee (sensitivity increase), this would result in the trade off of misclassifying a patient as a non-absconder/ nonescapee, resulting in an overlooked escape (specificity decrease) and in a cost of: $61 \times 0+8 \times 0+7 \times 20+7 \times$ $100=840$ units. If we now turn these considerations around and increase specificity with a loss of sensitivity, i.e., identify absconder/escapees with more efficiency, this results in a cost of: $59 \times 0+10 \times 0+9 \times 20+5 \times$ $100=680$ units. In summary, a decrease in specificity leads to an increase in undetected absconders/ escapees and a massive cost. In other words, for one missed absconder/ escapee, five patients may be erroneously treated too restrictively. Thus, these considerations show that detection of actual absconders and escapees are 


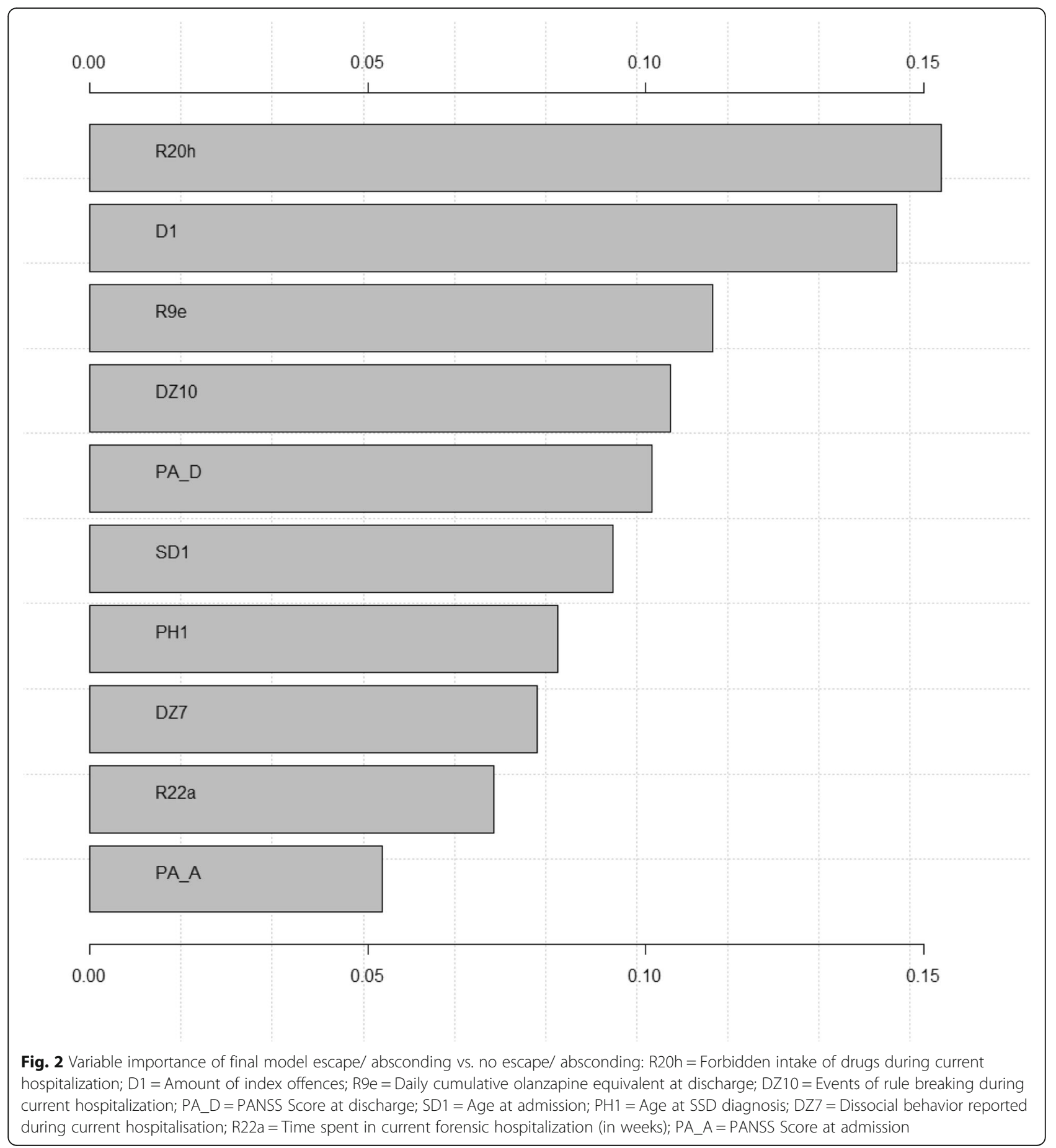

essential for model building. It also demonstrates that unbalanced data can lead to costly consequences. But it is not only these statistical considerations that must be considered when developing a potential tool. How expensive is it to treat a patient too restrictively, for example, and how much more expensive is it to have a patient drop out and potentially commit new crimes? At what level can we talk about acceptable risk and what is the minimum level of sensitivity and specificity that must be achieved? Rather complex questions that affect different areas like health and security policy, ethics must be addressed when developing and using a tool for risk assessment.

The results of the present explorative study may, nevertheless, provide benefits for clinical practice. By identifying a potentially problematic subgroup early in 
the course of hospitalization, the needs and risks of said subgroup can be addressed more effectively. A more individualized treatment of symptoms and an expansion of therapy to address dissocial behavioral patterns could possibly reduce the rate of escape and absconding.

However, since most of the risk assessment tools employed today serve as the basis for a variety of decisions about effective punishment and treatment [86], care must be taken to ensure that patients are not stigmatized or receive inferior treatment due to certain parameters like race or gender. Courts use such tools to assess the likelihood of recidivism or escape of pretrial detainees or offenders in bail and probation proceedings, or to set bail amounts [87]. ML methods tend to shift the trend of risk assessment toward prediction rather than intervention, as prediction-oriented risk assessments do not take into account the criminogenic nature of the criminal justice system itself, although the socially useful purpose of risk assessments should be directed more toward their utility as diagnostic and treatment guidance tools [86]. Bias can enter the design of a statistical model as well as the interpretation since the interpreter may more or less unconsciously incorporate personal political orientations, values and opinions when interpreting results [87]. In line with other authors [8688], we emphasize the importance of providing decision makers, such as judges, with additional information about the limitations immanent in risk score predictions and encourage further efforts to better understand how risk assessment tools interact with biases and beliefs held by the individuals who rely on them.

In conclusion, although the performance parameters of our model seem to be reasonable, this sensitive topic with consequences for the medical treatment of humans is far from being an automated machine selection process. To achieve this, high sensitivity and specificity rates would be required. In this study, ML must be seen as an advanced statistical method for retrospective differentiation of individuals rather than a predictive modeling technique.

\section{Limitations}

In addition to the well-known difficulties of retrospective data analysis (e.g., information loss), the database of 370 patients may be large for this specific group of offender patients with SSD, but is rather small for ML. This is accentuated by the fact that the group of patients who escaped or absconded was even smaller and artificially manipulated through imputation and balancing. Unbalanced data may cause algorithms to misrepresent the distributional properties of the data and lead to biased accuracies - this could be a problem in interpreting our final validation model. Previous research on the one hand recommends modeling with balanced data [89], and on the other hand advises against full artificial balancing as this can lead to overfitting [90]. Since we have chosen to balance the model as much as possible in the nested modeling, and at the same time significantly reducing the performance values of the model during validation, we have to consider overfitting in the modeling process. As this is the first ML model on this topic and a very specific population, further studies should test and validate this model on other (bigger and more balanced) patient populations, possibly employing a prospective study design and compare ML outcomes with clinical impressions of staff, to better assess true performance measures. This may help to identify other influencing factors and ultimately increase sensitivity and specificity. However, future studies may use the results of the present study, allowing more patients and fewer variables to be evaluated in order to improve the use of resources and build stronger models.

\section{Conclusion}

For the first time it was possible to create and describe a model using innovative ML analysis to identify influential factors related to escape and absconding in forensic patients with SSD. Patients who escaped or absconded were generally younger and showed more pronounced pathological manifestations. They exhibited an overall more problematic and rule-breaking behavior. Early identification of these patients could help to prevent problematic events from occurring in the first place by providing specifically tailored treatment strategies for these patients.

\section{Abbreviations}

SSD: Schizophrenia spectrum disorder; PANSS: Positive and Negative Syndrome Scale; NHST: Null hypothesis significance testing; ICD: International Classification of Diseases; SD: Standard deviation; ML: Machine Learning; AUC: Area Under the Curve; PPV: Positive Predictive Value; NPV: Negative Predictive Value

\section{Supplementary Information}

The online version contains supplementary material available at https://doi. org/10.1186/s12888-021-03117-1.

\section{Additional file 1}

Acknowledgements

Not applicable.

\section{Authors' contributions}

JK and SL designed the study and protocol. The survey of the data via protocol was performed independently by both JK and SL. All statistical analyses were carried out by JK. The first draft of the manuscript was done by JK. MS and SL edited multiple drafts, the revision and supervised the statistical analyses. All authors read and approved the final version of the manuscript.

Funding

No funding. 


\section{Availability of data and materials}

The datasets generated and/or analysed during the current study are not publicly available due to the sensitive nature of the data concerning patients in a forensic hospital but are available from the corresponding author on reasonable request.

\section{Declarations}

\section{Ethics approval and consent to participate}

This study was reviewed and approved by the Ethics Committee Zurich [Kanton Zürich] (committee's reference number: KEK-ZH-NR 2014-0480) as a retrospective anonymised observational data analysis. Informed consent was obtained either from the patients hospitalised during the study or their guardian/legally authorized representative prior to analysis. No patients were under the age of 16 years, therefore no written informed consent from a parent or guardian was required. The study complied with the Helsinki Declaration of 1975, revised in 2008.

\section{Consent for publication}

Not applicable.

\section{Competing interests}

All authors declare that they have no competing interests.

Received: 21 October 2020 Accepted: 12 February 2021

Published online: 04 March 2021

\section{References}

1. Chaplin E, Hearn D, Ndegwa D, Norman P, Hammond N. Developing the leave/abscond risk assessment (LARA) from the absconding literature: an aide to risk management in secure services. Adv Ment Heal Intellect Disabil. 2012;6:280-90

2. Mezey G, Durkin C, Dodge L, White S. Never ever? Characteristics, outcomes and motivations of patients who abscond or escape: a 5-year review of escapes and absconds from two medium and low secure forensic units. Crim Behav Ment Health. 2015;25:440-50.

3. Cullen AE, Jewell A, Tully J, Coghlan S, Dean K, Fahy T. A prospective cohort study of absconsion incidents in forensic psychiatric settings: can we identify those at high-risk? PLoS One. 2015;10:e0138819.

4. Stewart $D$, Bowers $L$. Absconding from psychiatric hospitals: a literature review. London Inst Psychiatry King's Coll London. 2010;1-43.

5. James R, Maude P. A focus on absconding in mental health: a review of the literature. Int J Heal Sci Res. 2015;5:400-9.

6. Bland RC, Parker JH. Some features of mental hospital elopements. Can Psychiatr Assoc J. 1974;19:463-7.

7. Molnar G, Keitner L, Swindall L. Medicolegal problems of elopement from psychiatric units. J Forensic Sci. 1985;30:44-9.

8. Walsh E, Rooney S, Sloan D, McCauley P, Mulvaney F, O'Callaghan E, et al. Irish psychiatric absconders: characteristics and outcome. Psychiatr Bull. 1998;22:351-3

9. Bowers $L$, Jarrett M, Clark N. Absconding: a literature review. J Psychiatr Ment Health Nurs. 1998;5:343-53.

10. Dickens $G L$, Campbell J. Absconding of patients from an independent UK psychiatric hospital: a 3-year retrospective analysis of events and characteristics of absconders. J Psychiatr Ment Health Nurs. 2001;8:543-50.

11. Falkowski J, Watts V, Falkowski W, Dean T. Patients leaving hospital without the knowledge or permission of staff-absconding. Br J Psychiatry. 1990;156: 488-90.

12. Lewis $A B \mathrm{Jr}$, Kohl RN. The risk and prevention of abscondence from an open psychiatric unit. Compr Psychiatry. 1962;3:302-8.

13. Meehan T, Morrison P, McDougall S. Absconding behaviour: an exploratory investigation in an acute inpatient unit. Aust New Zeal J Psychiatry. 1999;33: 533-7.

14. Milner G. The absconder. Compr Psychiatry. 1966;7(3):147-51.

15. Kernodle RW. Nonmedical leaves from a mental hospital. Psychiatry. 1966; 29:25-41.

16. Huber CG, Schneeberger AR, Kowalinski E, Fröhlich D, von Felten S, Walter $\mathrm{M}$, et al. Suicide risk and absconding in psychiatric hospitals with and without open door policies: a 15 year, observational study. Lancet Psychiatry. 2016;3:842-9.
17. Muir-Cochrane E, Oster C, Grotto J, Gerace A, Jones J. The inpatient psychiatric unit as both a safe and unsafe place: implications for absconding. Int J Ment Health Nurs. 2013;22:304-12.

18. Huws $R$, Shubsachs A. A study of absconding by special hospital patients: 1976 to 1988. J Forensic Psychiatry. 1993;4:45-58.

19. Brook R, Dolan M, Coorey P. Absconding of patients detained in an English special hospital. J Forensic Psychiatry. 1999;10:46-58.

20. Smith J, Quaynor E. Absconding from a regional secure unit. J Forensic Psychiatry. 1990;1:245-50.

21. Dolan M, Snowden P. Escapes from a medium secure unit. J Forensic Psychiatry. 1994;5:275-86.

22. Short J. Characteristics of absconders from acute admission wards. J forensic Psychiatry. 1995;6:277-84.

23. Wilkie T, Penney SR, Fernane S, Simpson AIF. Characteristics and motivations of absconders from forensic mental health services: a case-control study. BMC Psychiatry. 2014;14:91.

24. Martin K, McGeown M, Whitehouse M, Stanyon W. Who's going to leave? An examination of absconding events by forensic inpatients in a psychiatric hospital. J Forens Psychiatry Psychol. 2018;29:810-23.

25. Simpson AIF, Penney SR, Fernane S, Wilkie T. The impact of structured decision making on absconding by forensic psychiatric patients: results from an AB design study. BMC Psychiatry. 2015;15:103.

26. Bowers $L$, Jarrett M, Clark N, Kiyimba F, McFarlane L. Absconding: outcome and risk. J Psychiatr Ment Health Nurs. 1999;6:213-8.

27. Bowers L, Jarrett M, Clark N, Kiyimba F, McFarlane L. Determinants of absconding by patients on acute psychiatric wards. J Adv Nurs. 2000;32: 644-9.

28. Bowers L, Jarrett M, Clark N, Kiyimba F, McFarlane L. 1. Absconding: why patients leave. J Psychiatr Ment Health Nurs. 1999;6:199-205.

29. Bowers L, Alexander J, Gaskell C. A trial of an anti-absconding intervention in acute psychiatric wards. J Psychiatr Ment Health Nurs. 2003;10:410-6.

30. Farragher B, Gannon M, Ahmad I. Absent without leave-can we predict those who go AWOL? Ir J Psychol Med. 1996;13:28-30.

31. Quinsey VL, Coleman G, Jones B, Altrows IF. Proximal antecedents of eloping and reoffending among supervised mentally disordered offenders. J Interpers Violence. 1997;12:794-813.

32. Mosel KA, Gerace A, Muir-Cochrane E. Retrospective analysis of absconding behaviour by acute care consumers in one psychiatric hospital campus in Australia. Int J Ment Health Nurs. 2010;19:177-85.

33. Gerace A, Oster C, Mosel K, O'Kane D, Ash D, Muir-Cochrane E. Five-year review of absconding in three acute psychiatric inpatient wards in a ustralia. Int J Ment Health Nurs. 2015;24:28-37.

34. Bowers $L$. The expression and comparison of ward incident rates. Issues Ment Health Nurs. 2000;21:365-74.

35. Muir-Cochrane E, Mosel KA. Absconding: a review of the literature 19962008. Int J Ment Health Nurs. 2008;17:370-8.

36. Tomison AR. Characteristics of psychiatric hospital absconders. Br J Psychiatry. 1989;154:368-71.

37. Muir-Cochrane E, Mosel K, Gerace A, Esterman A, Bowers L. The profile of absconding psychiatric inpatients in Australia. J Clin Nurs. 2011;20(5-6):706-13.

38. Cabarkapa S, Sadhu R, King J, Dowling N, Radhakrishnan R, Akinbiyi A, et al. Absconding from Public Mental Health Inpatient Units-Who Does it, and why? Psychiatr Q. 2021;92:229-37.

39. Andoh B. Selected characteristics of absconders and non-absconders from mental hospitals: a comparison. Int J Soc Psychiatry. 1999:45:117-24.

40. Nijman H, Bowers L, Haglund K, Muir-Cochrane E, Simpson A, Van Der Merwe M. Door locking and exit security measures on acute psychiatric admission wards. J Psychiatr Ment Health Nurs. 2011;18:614-21.

41. Verma DK, Khanra S, Goyal N, Das B, Khess CRJ, Munda SK, et al. Absconding during inpatient care from a tertiary psychiatric hospital: a comparative study. Indian J Psychol Med. 2020;42(5):0253717620929182.

42. Moore E, Hammond S. When statistical models fail: problems in the prediction of escape and absconding behaviour from high-security hospitals. J Forensic Psychiatry. 2000;11:359-71.

43. Field A. Discovering statistics using IBM SPSS statistics. London: Sage; 2013.

44. Sainani KL. The problem of multiple testing. PM\&R. 2009;1:1098-103.

45. Perneger TV. What's wrong with Bonferroni adjustments. Bmj. 1998;316: 1236-8.

46. Benjamini Y, Hochberg Y. Controlling the false discovery rate: a practical and powerful approach to multiple testing. J R Stat Soc Ser B. 1995;57:289300. 
47. Witten J, Hastie D, Tibshirani R. An introduction to statistical learning with applications in R. New York: Springer; 2013.

48. Bzdok D, Meyer-Lindenberg A. Machine learning for precision psychiatry: opportunities and challenges. Biological Psychiatry: Cognitive Neuroscience and Neuroimaging. 2018;3:223-30.

49. Dwyer DB, Falkai $P$, Koutsouleris N. Machine learning approaches for clinical psychology and psychiatry. Annu Rev Clin Psychol. 2018;14:91-118.

50. Gillan CM, Whelan R. What big data can do for treatment in psychiatry. Curr Opin Behav Sci. 2017;18:34-42.

51. Crichton JHM. Defining high, medium, and low security in forensic mental healthcare: the development of the matrix of security in Scotland. J Forens Psychiatry Psychol. 2009;20:333-53.

52. World Health Organization (WHO). ICD-9: International classification of diseases (9th revision). Geneva: World Health Organization; 1978.

53. World Health Organization (WHO). ICD-10: International statistical classification of diseases and related health problems: Tenth revision. 5th edition. Geneva: World Health Organization; 2016.

54. Kirchebner J, Günther MP, Sonnweber M, King A, Lau S. Factors and predictors of length of stay in offenders diagnosed with schizophrenia- a machine-learning-based approach. BMC Psychiatry. 2020;20:1-12.

55. Kirchebner J, Sonnweber M, Nater UM, Günther M, Lau S. Stress, schizophrenia, and violence: a machine learning approach. J Interpers Violence. 2020.

56. Bischl B, Lang M, Kotthoff L, Schiffner J, Richter J, Studerus E, et al. Mlr: machine learning in R. J Mach Learn Res. 2016;17:5938-42.

57. Brodersen KH, Ong CS, Stephan KE, Buhmann JM. The balanced accuracy and its posterior distribution, Proceedings - International Conference on Pattern Recognition; 2010. p. 3121-4.

58. Ishwaran $H$, Kogalur UB. RandomForestSRC: Fast unified random forests for survival, regression, and classification (RF-SRC). R package, version 2.9. 3; 2020.

59. James G, Witten D, Hastie T, Tibshirani R. An introduction to statistical learning. New York: Springer; 2013.

60. Campbell G. Advances in statistical methodology for the evaluation of diagnostic and laboratory tests. Stat Med. 1994;13:499-508.

61. Moons KGM, de Groot JAH, Bouwmeester W, Vergouwe Y, Mallett S, Altman DG, et al. Critical appraisal and data extraction for systematic reviews of prediction Modelling studies: the CHARMS checklist. PLoS Med. 2014;11.

62. Studerus E, Ramyead A, Riecher-Rössler A. Prediction of transition to psychosis in patients with a clinical high risk for psychosis: a systematic review of methodology and reporting. Psychol Med. 2017:47:1163-78.

63. Browne MW. Cross-validation methods. J Math Psychol. 2000;44:108-32.

64. Cortez P, Embrechts MJ. Using sensitivity analysis and visualization techniques to open black box data mining models. Inf Sci (Ny). 2013;225:117.

65. Leucht S, Samara M, Heres S, Patel MX, Furukawa T, Cipriani A, et al. Dose equivalents for second-generation antipsychotic drugs: the classical mean dose method. Schizophr Bull. 2015;41:1397-402.

66. Leucht S, Samara M, Heres S, Patel MX, Woods SW, Davis JM. Dose equivalents for second-generation antipsychotics: the minimum effective dose method. Schizophr Bull. 2014;40:314-26.

67. Gardner DM, Murphy AL, O'Donnell H, Centorrino F, Baldessarini RJ. International Consensus Study of Antipsychotic Dosing. Focus (Madison). 2014;12:235-43.

68. Bonta J, Blais J, Wilson HA. A theoretically informed meta-analysis of the risk for general and violent recidivism for mentally disordered offenders. Aggress Violent Behav. 2014;19:278-87.

69. Hodgins S, Piatosa MJ, Schiffer B. Violence among people with schizophrenia: phenotypes and neurobiology. In: Miczek K, MeyerLindenberg A, editors. Neuroscience of aggression. New York: Springer; 2014. p. 329-68

70. Kooyman I, Walsh E, Stevens H, Burns T, Tyrer P, Tattan T, et al. Criminal offending before and after the onset of psychosis: examination of an offender typology. Schizophr Res. 2012;140:198-203.

71. Lau S, Günther MP, Kling S, Kirchebner J. Latent class analysis identified phenotypes in individuals with schizophrenia spectrum disorder who engage in aggressive behaviour towards others. Eur Psychiatry. 2019;60:8696.

72. Simpson Al, Grimbos T, Chan C, Penney SR. Developmental typologies of serious mental illness and violence: evidence from a forensic psychiatric setting. Aust N Z J Psychiatry. 2015;49:1048-59. https://doi.org/10.1177/0004 867415587745

73. Tengström A, Hodgins S, Kullgren G. Men with schizophrenia who behave violently: the usefulness of an early-versus late-start offender typology. Schizophr Bull. 2001;27:205-18.

74. van Dongen J, Buck N, Van Marle H. Unravelling offending in schizophrenia: factors characterising subgroups of offenders. Crim Behav Ment Health. 2015;25:88-98.

75. Witt K, van Dorn R, Fazel S. Risk factors for violence in psychosis: systematic review and meta-regression analysis of 110 studies. PLoS One. 2013;8: e55942. https://doi.org/10.1371/journal.pone.0055942 Accessed 18 Apr 2019.

76. Elbogen EB, Johnson SC. The intricate link between violence and mental disorder: results from the national epidemiologic survey on alcohol and related conditions. Arch Gen Psychiatry. 2009;66:152-61.

77. Wallace C, Mullen PE, Burgess P. Criminal offending in schizophrenia over a 25-year period marked by deinstitutionalization and increasing prevalence of comorbid substance use disorders. Am J Psychiatry. 2004;161:716-27.

78. Appelbaum PS, Robbins PC, Monahan J. Violence and delusions: data from the MacArthur violence risk assessment study. Am J Psychiatry. 2000;157: 566-72.

79. Douglas KS, Guy LS, Hart SD. Psychosis as a risk factor for violence to others: a meta-analysis. Psychol Bull. 2009:135:679-706.

80. Link BG, Andrews H, Cullen FT. The violent and illegal behavior of mental patients reconsidered. Am Sociol Rev. 1992;57:275.

81. Mojtabai R. Psychotic-like experiences and interpersonal violence in the general population. Soc Psychiatry Psychiatr Epidemiol. 2006:41:183-90.

82. Steadman HJ, Mulvey EP, Monahan J, Robbins PC, Appelbaum PS, Grisso T, et al. Violence by people discharged from acute psychiatric inpatient facilities and by others in the same neighborhoods. Arch Gen Psychiatry. 1998;55:393-401.

83. Swanson JW, Swartz MS, Van Dorn RA, Elbogen EB, Wagner HR, Rosenheck RA, et al. A national study of violent behavior in persons with schizophrenia. Arch Gen Psychiatry. 2006;63:490-9.

84. Swanson JW, Van Dorn RA, Swartz MS, Smith A, Elbogen EB, Monahan J. Alternative pathways to violence in persons with schizophrenia: the role of childhood antisocial behavior problems. Law Hum Behav. 2008;32:228-40.

85. Taylor PJ. Motives for offending among violent and psychotic men. Br J Psychiatry. 1985;147:491-8.

86. Barabas C, Dinakar K, Ito J, Virza M, Zittrain J, Friedler SA, et al. Interventions over predictions: reframing the ethical debate for actuarial risk assessment. PMLR; 2018.

87. Završnik A. Algorithmic justice: Algorithms and big data in criminal justice settings. Eur J Criminol. 2019;00(0):1-20.1477370819876762.

88. Angwin J, Larson J, Mattu S, Kirchner L. Machine bias: There's software used across the country to predict future criminals. it's biased against blacks ProPublica; 2016. p. 23

89. Wei $Q$, Dunbrack RL Jr. The role of balanced training and testing data sets for binary classifiers in bioinformatics. PLOS One. 2013:8:e67863.

90. He H, Garcia EA. Learning from imbalanced data. IEEE Trans Knowl Data Eng. 2009:21:1263-84.

\section{Publisher's Note}

Springer Nature remains neutral with regard to jurisdictional claims in published maps and institutional affiliations.

\section{Ready to submit your research? Choose BMC and benefit from:}

- fast, convenient online submission

- thorough peer review by experienced researchers in your field

- rapid publication on acceptance

- support for research data, including large and complex data types

- gold Open Access which fosters wider collaboration and increased citations

- maximum visibility for your research: over $100 \mathrm{M}$ website views per year

At BMC, research is always in progress.

Learn more biomedcentral.com/submission 